Additional Comments on

\title{
IMPEDANCE MEASUREMENTS AND STANDARDS*
}

\section{G. A. Deschamps}

Investigations of magnified and squared SWR responses and the use of auxiliary tuners with directional couplers both led to a modified reflectometer technique permitting accuracies of better than 0.1 percent in SWR measurements [Beatty, 1958].

The use of auxiliary tuners with a directional coupler also permits the construction of a highly accurate phase shifter. Combination of this with the above modified reflectometer yields an impedance meter similar to a bridge and both magnitude and phase of the reflection coefficient may be independently determined to high accuracy [Engen, 1959].

Possible errors in the use of a sliding short-circuit as a phase shift standard have been discussed and a method for avoiding them described. Accuracies of 0.3 degree have been obtained [Magid, 1958].

Techniques for the measurement of the equivalent network parameters of discontinuities coupling two or more modes in multimode waveguides have been described [Felsen et al., 1959; Lewis, 1959].

Measurement of standing waves in a specially constructed line was used to evaluate high dielectric constants at UHF [Williams and Foster, 1957].

Rapid measurement of impedance or transmission properties over a wide range of frequencies continues to be of great interest. A system for measuring phase and attenuation through a component [Linker and Grimm, 1958] from 8.7 to $9.6 \mathrm{kMc} / \mathrm{s}$ and an automatic impedance plotter [Watts and Alford, 1957] covering the frequency ranges 180 to $900 \mathrm{Mc} / \mathrm{s}$ and 50 to $250 \mathrm{Mc} / \mathrm{s}$ were developed.

Automatic continuous measurement of phase at microwave can be done by shifting the frequency to audiofrequency [Mittra, 1957]. A broadband direct reading measuring device has been constructed where the LO tracts the $\mathrm{RF}$ in order to produce a fixed intermediary frequency [Dropkin, 1958].

*This material belongs after the fourth paragraph of the published report (p. 598, J. Research NBS, 64D, 6,) and must be used with the references on that page. 